\title{
Identification of Genes Differentially Expressed in Mouse Fetuses from Streptozotocin-induced Diabetic Pregnancy by cDNA Subtraction
}

\author{
NANAKO SATO*,**, YoshIHISA SUGIMURA*,***, YOSHITAKA HAYASHI*, TAKASHI MURASE*, YASUHIKO \\ KANOU*,\#, FUMITAKA KIKKAWA** AND YOSHIHARU MURATA* \\ *Department of Genetics, Research Institute of Environmental Medicine, Nagoya University, Nagoya 464-8601, Japan \\ **Department of Obstetrics and Gynecology, Nagoya University Graduate School of Medicine, Nagoya 466-8550, Japan \\ ***Department of Endocrinology and Diabetes, Nagoya University Graduate School of Medicine, Nagoya 466-8550, Japan \\ \#Futuristic Environmental Simulation Center, Research Institute of Environmental Medicine, Nagoya University, Nagoya 464-8601, Japan
}

\begin{abstract}
Epidemiological studies have shown that the risks of fetal malformation such as neural tube defects increase in diabetic pregnancy. To explore the mechanism of fetal malformation induced by diabetes, cDNA subtraction using mouse embryos (E9.5) of diabetic dams and those of controls was performed to identify differentially expressed genes. The expression level of genes identified by cDNA subtraction was further verified by quantitative RT-PCR using E8.5 embryos, and differential expression of 4 genes, Brcc3, Commd3, Ddx1, and SET was confirmed. We also analyzed the expression level of neural tube defect-related genes, and found that Folbp1, EphrinA5 and Sox10 were differentially expressed. Altered expression of these genes mostly persisted throughout the later stages of the development (E10.514.5). Hierarchical clustering analysis showed correlation between expression levels of these genes, suggesting that these genes cooperatively play a role in embryonic development. Our results suggest that an altered gene expression profile in embryos underlies the development of congenital malformation in diabetic pregnancies.
\end{abstract}

Key words: Congenital malformation, Neural tube defect, cDNA subtraction, Gene expression profile, Embryonic development

(Endocrine Journal 55: 317-323, 2008)

EPIDEMIOLOGICAL studies have shown that the risks of fetal malformations and spontaneous abortions increase in diabetic pregnancy $[1,2]$. In recent studies, the incidence of congenital malformation in type 1 diabetic pregnancy was estimated to be $4-10 \%[3-5]$. The malformations associated with diabetic pregnancy involve many major organ systems, such as the central nervous, cardiovascular, gastrointestinal, urogenital and musculoskeletal systems [4, 5]. Such malformations have been reproduced in rodent models for diabetic pregnancy, in which maternal hyperglycemia is

Received: July 10, 2007

Accepted: January 4, 2008

Correspondence to: Dr. Yoshitaka HAYASHI, M.D., Ph.D., Department of Genetics, Research Institute of Environmental Medicine, Nagoya University, Nagoya 464-8601, Japan induced by streptozotocin-induced destruction of pancreatic beta cells [6-8]. Among various malformations, neural tube defects (NTDs) can be recognized at the earlier phase of pregnancy, thereby serving as an index for congenital malformation.

NTDs in fetus have been reported not only in animal models for diabetic pregnancies, but also in those with a number of genetic abnormalities listed in a mouse genome informatics database $[9,10]$, indicating that coordinated expression of numerous genes is required for neural tube closure. Thus, discordant expression of various genes may also result in NTDs by diabetes. However, only limited number of genes, such as Pax3 $[7,11]$ and folate binding protein 1 (Folbp1) [12], has been analyzed in these models.

In the present study, we applied cDNA subtraction to identify genes that are differentially expressed in 
mouse embryos of diabetic dams from those of the controls. We also screened the expression of genes that have been reported to be involved in NTDs. By these approaches, we newly identified 6 genes that were differentially expressed. Our results should support the hypothesis that an altered gene expression program in embryos underlies the development of congenital malformation in diabetic pregnancies.

\section{Materials and Methods}

\section{Animal procedures and RNA extraction}

The animal experiment protocol conformed to the NIH animal care guideline and was approved by our Institutional Animal Care and Use committee in accordance with guidelines for animal care in Nagoya University. Female ICR mice (6-7 weeks old) weighing approximately $24 \mathrm{~g}$ were purchased from Japan SLC Inc. (Hamamatsu, Japan). They were housed in a specific pathogen free animal facility under conditions of constant temperature $\left(23^{\circ} \mathrm{C}\right), 12$-h/12-h light/dark cycle, and free access to standard chow and tap water. The mice were rendered hyperglycemic by an intraperitoneal injection of streptozotocin (STZ, $240 \mathrm{mg} / \mathrm{kg}$ body weight: Sigma Chemical Co., St. Louis, MO) resolved in citrate-buffered saline. Control mice were injected with vehicle. Seven days after the STZ injection, peripheral blood glucose concentration was measured using a compact glucose analyzer (MediSafe, Terumo, Tokyo, Japan). Diabetic state was defined as a glucose concentration exceeding $400 \mathrm{mg} / \mathrm{dl}$. Diabetic female mice (DM) were mated overnight with non-diabetic male ICR mice. The morning when a vaginal plug was found was referred to as embryonic day 0.5. On the planned days (E8.5, E9.5, E10.5, E12.5, E14.5) the mice were sacrificed and embryos were extracted. For E8.5, fifteen embryos were extracted from 3 control dams (5 embryos from each dam). The same number of embryos was extracted from 5 diabetic dams ( 3 embryos from each dam). For, E10.5-14.5, six embryos from 2 diabetic dams (3 embryos from each dam) and, 3 embryos from a control dam were extracted. Embryos that showed bleeding in placenta, which were often found in diabetic dams, were excluded from the study. Total RNA was extracted from whole embryos of E8.5-9.5, and E10.5-14.5 head using RNeasy kits (Qiagen, Valencia, CA) according to the manufacturer's protocol.

\section{cDNA subtraction}

Total RNA extracted from E9.5 embryos were used for cDNA subtraction by using CLONTECH PCR-Select cDNA Subtraction Kit and BD SMART mRNA Amplification Kit according to the manufacturer's protocol [13]. Briefly, 2 embryos that showed NTD from a diabetic dam were subjected for RNA extraction and extracted RNA preparations were used for cDNA synthesis. From 2 embryos from a control dam, cDNAs were also prepared in the same manner. Both forward and reverse subtraction was performed and amplified cDNA fragments were cloned into pGEM-T easy plasmid (Promega, Madison, MI). The cloned genes were screened for possible differential expression by using CLONTECH PCR-Select Differential Screening Kit according to the manufacturer's protocol. Sequences of the clones that passed the differential screening were verified and the sequence was subjected to BLAST search to identify whole gene sequence.

\section{Real time RT-PCR}

One microgram of total RNA was reverse-transcribed into cDNA and cDNAs equivalent to $20 \mathrm{ng}$ of total RNA were used for PCR. Real-time monitored quantitative PCR was carried out using ABI-PRISM 7000 (Applied Biosystems, Foster City, CA) and the Power SYBR Green Master Mix (Applied Biosystems). Primer pairs to amplify specific cDNAs were designed to amplify 150-200 bp cDNA fragments, except for glyceraldehyde-3-phosphate dehydrogenase (GAPDH) cDNA [14]. The sequences of the primers are summarized in Table 1. Gene expression level was normalized by that of GAPDH and expressed in an arbitrary unit in which the mean of the control was defined as 1.00. Detailed procedure has been described previously [14].

\section{Statistical analyses}

Unless otherwise stated, results were expressed as means \pm SE. Statistical analyses were performed using a Student's $t$-test using StatView software. $\mathrm{P}$ values less than 0.05 were considered to be significant. Correlation between expression level of genes was analyzed by Pearson product-moment correlation coefficient 
Table 1. primers used for quantitative RT-PCR

\begin{tabular}{|c|c|c|c|}
\hline Gene & sense/antisense & sequence & size of amplicons (bp) \\
\hline \multirow[t]{2}{*}{ brec3 } & sense & ggactcagtgaccaagatcc & \\
\hline & antisense & gttctcatgctttattctagggaag & 195 \\
\hline \multirow[t]{2}{*}{ commd3 } & sense & catacttgaaacagatcgac & \\
\hline & antisense & caaaacagttctattcgctc & 158 \\
\hline \multirow[t]{2}{*}{ ddx 1} & sense & aagccetcttccetgectgt & \\
\hline & antisense & agcttttggagcattcgggaga & 194 \\
\hline \multirow[t]{2}{*}{ ephrinA5 } & sense & gacattatagcacagtctcctcccgt & \\
\hline & antisense & cccaagttgttgcttagaaatcagg & 171 \\
\hline \multirow[t]{2}{*}{ folbp 1} & sense & ggccaggttttctgggettt & \\
\hline & antisense & agtcatcaggtgagccatgtc & 157 \\
\hline \multirow[t]{2}{*}{ gapdh } & sense & gcaaagtggagattgttgccat & \\
\hline & antisense & gatgcagggatgatgttctgg & 553 \\
\hline \multirow[t]{2}{*}{ set } & sense & gtgtctgcactgettgggga & \\
\hline & antisense & gaagacgggtcaccactctca & 185 \\
\hline \multirow[t]{2}{*}{$\operatorname{sox} 10$} & sense & cagtccggcaaggcagaccc & \\
\hline & antisense & gcaggtattggtccagctcagtcac & 157 \\
\hline
\end{tabular}

method, as well as hierarchical clustering using Cluster 3.0 and Java TreeView softwares (downloaded from http://bonsai.ims.u-tokyo.ac.jp/ mdehoon/software/ cluster/software.htm\#ctv).

\section{Results and Discussion}

To identify genes differentially expressed in fetus of streptozotocin-induced diabetic dams, cDNA subtraction was performed using E9.5 embryos. This developmental stage was selected because neural tube closure proceeds during E8.5-9.5 (Theiler stage 13-14). Two embryos that showed NTD from a diabetic dam and two embryos from a control dam were used for cDNA synthesis, and cDNA subtraction was performed as described in Materials and Methods. The expression level of genes cloned by the subtraction was quantified by real-time RT-PCR using total RNA extracted from E8.5 whole embryos. Through these approaches, we identified 4 genes that were differentially expressed in embryos of diabetic dams compared to those of control dams (Fig. 1). These genes were, SET translocation (SET) [15], DEAD (Asp-Glu-Ala-Asp) box polypeptide 1 (Ddx1) [16], COMM domain containing 3 (Commd3) [17] and BRCA1/BRCA2-containing complex, subunit 3 (Brcc3) [18]. SET has been assumed to play a role in nucleosome assembly [15]. Brcc3 is a subunit included in BRCA complexes that are involved in DNA repair [19]. Ddx1 is a putative RNA helicase that binds hnRNP [20] and Commd3 is assumed to block NF-kB function via inhibiting access of NF-kB to chromatin [17]. We also quantified the expression level of genes that have been reported to be involved in neural crest/tube defects, and found that 3 genes, EphrinA5 [21], Folbp1 [22], and Sox10 [23], were differentially expressed (Fig. 1). Whereas NTDs have been reported in mice defective for the latter three genes [21-23], no gene-targeting animal model has ever been reported for SET, Commd3, Ddx1, and Brcc3.

Next, we analyzed the correlation among gene expression levels. Since gene expression patterns dramatically change as development proceeds, subtle

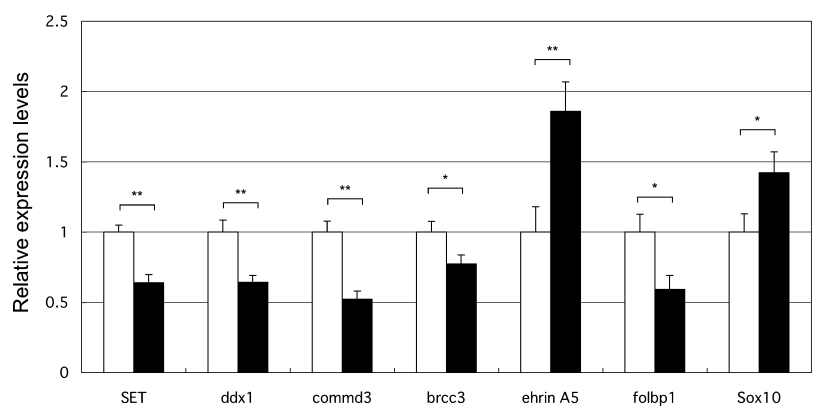

Fig. 1. Gene expression levels in embryos of diabetic or control dams.

Total RNA were extracted from E8.5 whole embryo and subjected to the gene expression level analyses. Open bars represent gene expression level in embryos of controls $(n=15)$ and close bars those of diabetic dams $(n=15)$. Results are expressed as mean $+/-$ SE relative to the controls. $*$ : p-value $<0.05, * *$ : p-vallue $<0.01$ 
fluctuation in developmental stage among embryos can affect gene expression patterns. Such fluctuation in developmental stage may be further enhanced in embryos of diabetic dams, as suggested by the fact that penetrance of NTDs in embryos of diabetic dams is not $100 \%[7,12]$. Thus, if the alteration in gene expression reflects the severity of influence of maternal hyperglycemia, severely affected fetus is expected to show larger alteration in gene expression. As shown in Fig. 2A and $\mathrm{B}$, a significantly positive correlation was observed between expression level of EphrinA5 and Sox10, as well as SET and Commd3. A significantly negative correlation was observed between expression levels of Folbp1 and Sox10 (Fig. 2C). The correlation among the gene expression levels with significance $(p<0.01)$, was schematically summarized in Fig. 2D, in which thickness of the lines represents the correlation coefficient. The correlation of gene expression levels was also analyzed using a hierarchical clustering method, and a similar result was obtained (Fig. 2E). These results suggest that these genes cooperatively play a role in embryonic development as well as in development of malformation in diabetic pregnancy.

We then analyzed the expression level of 7 genes in

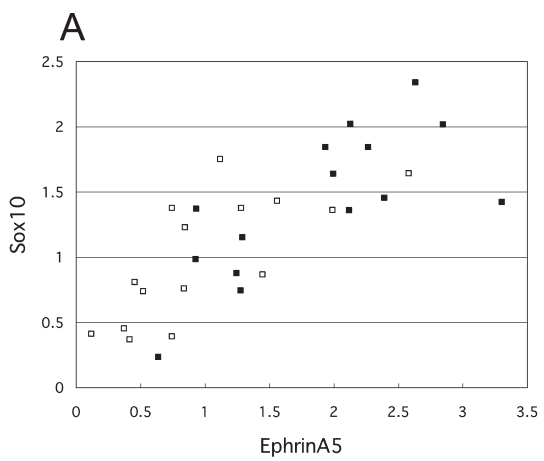

B

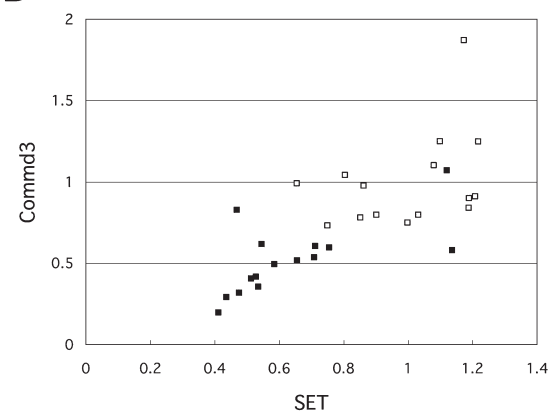

D

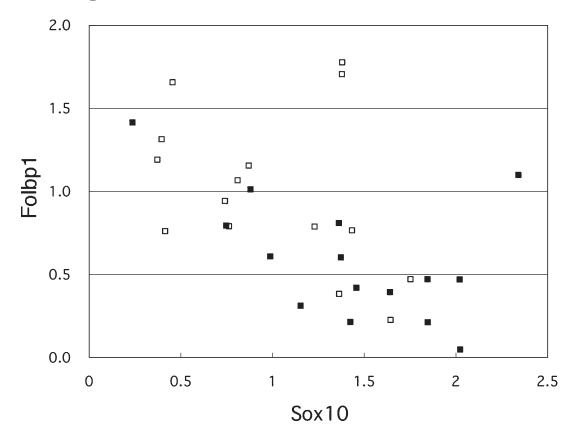

E

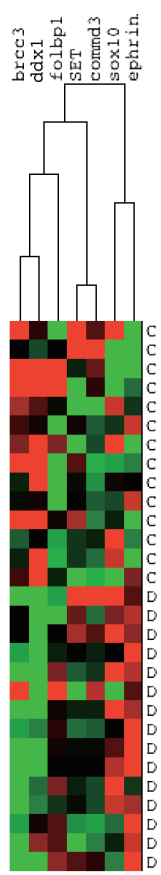

Fig. 2. Correlation of gene expression levels.

A) Correlation between expression levels of EphrinA5 and Sox 10

Open squares represent embryos of controls and closed squares those of diabetic dams.

B) Correlation between expression levels of SET and Commd3

Open squares represent embryos of controls and closed squares those of diabetic dams.

C) Correlation between expression levels of Folbp1 and Sox 10

Open squares represent embryos of controls and closed squares those of diabetic dams.

D) Schematic presentation of correlation among gene expression levels

Correlation between expression levels of genes were analyzed by Pearson product-moment correlation coefficient method, and the correlation among the gene expression levels with significance $(p<0.01)$, was schematically summarized. Black lines indicate positive correlation, whereas red lines with arrowheads indicate negative correlation. Thickness of the lines represents the correlation coefficient.

E) Hierarchical clustering analysis of gene expression pattern.

Hierarchical clustering were performed using Cluster 3.0 and Java TreeView (downloaded from http://bonsai.ims.utokyo.ac.jp/ mdehoon/software/cluster/software.htm\#ctv). Red colors represent higher expression levels, while green colors represent lower expression levels. Tree diagram on the top of the figure indicate the correlation between the gene expression levels. $\mathrm{C}$ and $\mathrm{D}$ at right margin indicate controls and fetuses from diabetic dams, respectively. 

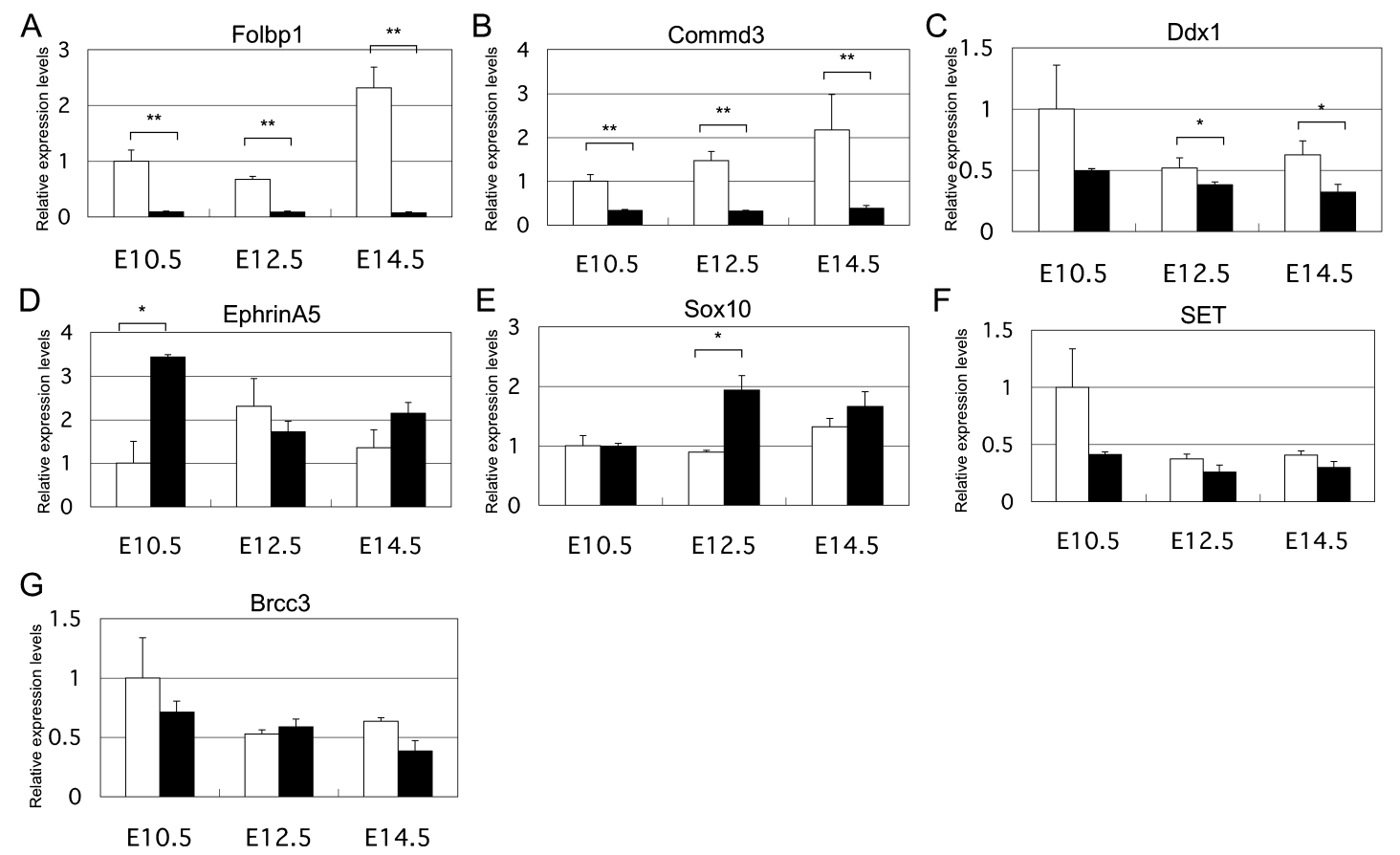

Fig. 3. Gene expression in the later stage of development in heads of embryos that are enriched in neural tube derived tissues.

Total RNA was extracted from the head part of the embryos at the indicated gestational dates. Open bars represent embryos of controls $(n=3)$ and close bars those of diabetic dams $(n=6)$. Results are expressed as mean $+/-$ SE relative to the expression level in the heads of E10.5 control embryos. *: p-value $<0.05, * *$ : p-vallue $<0.01$

later stages of embryonic development. For these analyses, we used the head of the E10.5-14.5 fetus, since the head is enriched with the tissues derived from neural tube. As shown in Fig. 3A and 3B, lower expressions of Folbp1 and Commd 3 in embryos of diabetic dams persisted throughout the observed periods. Lower expression of Ddx1 (Fig. 3C) as well as higher expression of EphrinA5 and Sox 10 (Fig. 3D and 3E) was observed in at least one developmental stage. Although the differences did not reach statistical significance, expression levels of SET and Brcc3 in fetuses from diabetic dams were mostly lower than the controls. Fifty percent of the embryos extracted from diabetic dams showed neural tube defects. However, no significant differences in expression level of these genes were observed between diabetic embryos showing NTD and diabetic embryos without NTD (data not shown). Thereby, the relation between the altered expression of these genes and development of malformation remains obscure. Nevertheless, it has been reported that the absence of Folbp1, EphrinA5 or Sox 10 results in NTD [21, 22, 23]. However, in the present study, expression of EphrinA5 and Sox 10 was higher in diabetic embryos. We may speculate that the increased expression of these two genes could be a protective response against the altered gene expression program caused by maternal diabetes. Interestingly, functional interaction between Sox10 and Pax3, which plays important roles in neural tube closure, has been reported [24].

Since lower expression of Commd 3 in embryos of diabetic dams persisted throughout the observed periods, we tested whether such differences are observed between adult diabetic mice brains and control. Expression levels in liver were also tested, as it is reported that Commd3 is expressed ubiquitously [17]. As shown in Fig. 4, significantly lower expression of Commd3 in brain, but not in liver, was observed. However, the difference in expression was far less pronounced compared to that in embryos, suggesting that regulatory mechanisms of Commd 3 expression in the embryos and adults are distinct.

In the present study we demonstrated that expression of multiple genes in embryos were altered in diabetic pregnancy. In the current approach, however, the organ or tissue in which the gene of interest is expressed remains unknown. Although we performed wholemount in situ hybridization for the genes identified 


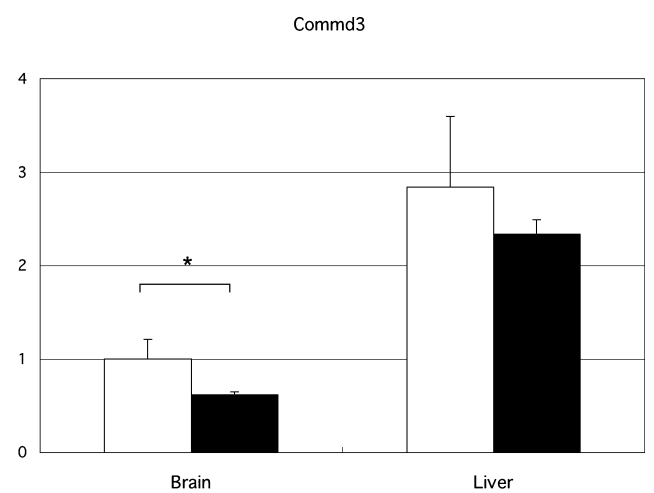

Fig. 4. Commd 3 expression in adult mice.

Total RNA was extracted from brains and livers of diabetic adult mice as well as from those from controls. Open bars represent control mice $(n=6)$ and close bars diabetic mice $(n=3)$. Results are expressed as mean $+/-$ $\mathrm{SE}$ relative to the expression level in the control. *: pvalue $<0.05$

herein, we could not observe specific expression patterns, probably due to the low expression levels of the identified genes. To further the understanding of the impact of maternal diabetes on regulatory program of gene expression in embryos, analysis of global gene expression profile with high-throughput methods such as cDNA microarray combined with micro-dissection technique should be performed. Such analyses would unveil the mechanism of fetal malformations in diabetic pregnancy and contribute to develop novel preventive countermeasures.

\section{Acknowledgements}

We are indebted to Dr. Mariko Itoh and Mr. Akihiko Takei in the animal care unit in the Futuristic Environmental Simulation Center in Research Institute of Environmental Medicine, Nagoya University for their help with the animal experiments. This work was supported in part by Grants-in-aid for Scientific Research from Japanese Society for the Promotion of Science 16590875 (to T. M.) and 18591019 (to Y. H.).

\section{References}

1. Miller E, Hare JW, Cloherty JP, Dunn PJ, Gleason RE, Soeldner JS, Kitzmiller JL (1981) Elevated maternal hemoglobin A1c in early pregnancy and major congenital anomalies in infants of diabetic mothers. $N$ Engl J Med 304: 1331-1334.

2. Eriksson UJ, Cederberg J, Wentzel P (2003) Congenital malformations in offspring of diabetic mothers - animal and human studies. Rev Endocr Metab Disord 4: 79-93.

3. Suhonen L, Hiilesmaa V, Teramo K (2000) Glycaemic control during early pregnancy and fetal malformations in women with type I diabetes mellitus. Diabetologia 43: 79-82.

4. Sheffield JS, Butler-Koster EL, Casey BM, McIntire DD, Leveno KJ (2002) Maternal diabetes mellitus and infant malformations. Obstet Gynecol 100: 925-930.

5. Evers IM, de Valk HW, Visser GH (2004) Risk of complications of pregnancy in women with type 1 diabetes: nationwide prospective study in the Netherlands. BMJ 328: 915.

6. Wentzel P, Thunberg L, Eriksson UJ (1997) Teratogenic effect of diabetic serum is prevented by supplementation of superoxide dismutase and $\mathrm{N}$-acetylcysteine in rat embryo culture. Diabetologia 40: 7-14.

7. Phelan SA, Ito M, Loeken MR (1997) Neural tube defects in embryos of diabetic mice: role of the Pax-3 gene and apoptosis. Diabetes 46: 1189-1197.
8. Loeken MR (2005) Current perspectives on the causes of neural tube defects resulting from diabetic pregnancy. Am J Med Genet C Semin Med Genet 135: 77-87.

9. Copp AJ (2005) Neurulation in the cranial regionnormal and abnormal. J Anat 207: 623-635.

10. Greene ND, Copp AJ (2005) Mouse models of neural tube defects: investigating preventive mechanisms. Am $J$ Med Genet C Semin Med Genet 135: 31-41.

11. Li R, Chase M, Jung SK, Smith PJ, Loeken MR (2005) Hypoxic stress in diabetic pregnancy contributes to impaired embryo gene expression and defective development by inducing oxidative stress. Am J Physiol Endocrinol Metab 289: E591-599.

12. Wentzel P, Gareskog M, Eriksson UJ (2005) Folic acid supplementation diminishes diabetes- and glucose-induced dysmorphogenesis in rat embryos in vivo and in vitro. Diabetes 54: 546-553.

13. Diatchenko L, Lau YF, Campbell AP, Chenchik A, Moqadam F, Huang B, Lukyanov S, Lukyanov K, Gurskaya N, Sverdlov ED, Siebert PD (1996) Suppression subtractive hybridization: a method for generating differentially regulated or tissue-specific cDNA probes and libraries. Proc Natl Acad Sci USA 93: 6025-6030.

14. Futaki S, Hayashi Y, Yamashita M, Yagi K, Bono H, Hayashizaki Y, Okazaki Y, Sekiguchi K (2003) Molecular basis of constitutive production of basement membrane components. Gene expression profiles of 
Engelbreth-Holm-Swarm tumor and F9 embryonal carcinoma cells. J Biol Chem 278: 50691-50701.

15. Fan Z, Beresford PJ, Oh DY, Zhang D, Lieberman J (2003) Tumor suppressor NM23-H1 is a granzyme Aactivated DNase during CTL-mediated apoptosis, and the nucleosome assembly protein SET is its inhibitor. Cell 112: 659-672.

16. Godbout R, Packer M, Bie W (1998) Overexpression of a DEAD box protein (DDX1) in neuroblastoma and retinoblastoma cell lines. J Biol Chem 273: 2116121168.

17. Burstein E, Hoberg JE, Wilkinson AS, Rumble JM, Csomos RA, Komarck CM, Maine GN, Wilkinson JC, Mayo MW, Duckett CS (2005) COMMD proteins, a novel family of structural and functional homologs of MURR1. J Biol Chem 280: 22222-22232.

18. Boudreau HE, Broustas CG, Gokhale PC, Kumar D, Mewani RR, Rone JD, Haddad BR, Kasid U (2007) Expression of BRCC3, a novel cell cycle regulated molecule, is associated with increased phospho-ERK and cell proliferation. Int J Mol Med 19: 29-39.

19. Farmer H, McCabe N, Lord CJ, Tutt AN, Johnson DA, Richardson TB, Santarosa M, Dillon KJ, Hickson I, Knights C, Martin NM, Jackson SP, Smith GC, Ashworth A (2005) Targeting the DNA repair defect in
BRCA mutant cells as a therapeutic strategy. Nature 434: 917-921.

20. Chen HC, Lin WC, Tsay YG, Lee SC, Chang CJ (2002) An RNA helicase, DDX1, interacting with poly(A) RNA and heterogeneous nuclear ribonucleoprotein K. J Biol Chem 277: 40403-40409.

21. Holmberg J, Clarke DL, Frisen J (2000) Regulation of repulsion versus adhesion by different splice forms of an Eph receptor. Nature 408: 203-206.

22. Piedrahita JA, Oetama B, Bennett GD, van Waes J, Kamen BA, Richardson J, Lacey SW, Anderson RG, Finnell RH (1999) Mice lacking the folic acid-binding protein Folbp1 are defective in early embryonic development. Nat Genet 23: 228-232.

23. Pingault V, Bondurand N, Kuhlbrodt K, Goerich DE, Prehu MO, Puliti A, Herbarth B, Hermans-Borgmeyer I, Legius E, Matthijs G, Amiel J, Lyonnet S, Ceccherini I, Romeo G, Smith JC, Read AP, Wegner M, Goossens M (1998) SOX10 mutations in patients with Waardenburg-Hirschsprung disease. Nat Genet 18: 171-173.

24. Bondurand N, Pingault V, Goerich DE, Lemort N, Sock E, Le Caignec C, Wegner M, Goossens M (2000) Interaction among SOX10, PAX3 and MITF, three genes altered in Waardenburg syndrome. Hum Mol Genet 9: 1907-1917. 
